\title{
Nonlinearity difference in the two passbands of a distributed-feedback semiconductor laser amplifier
}

\author{
Rongqing Hui and Adalberto Sapia \\ Fondazione Ugo Bordoni, Viale Europa 190, 00144 Rome, Italy
}

Received February 26, 1990; accepted June 13, 1990

\begin{abstract}
The nonlinearity difference in the two passbands of a distributed-feedback semiconductor laser amplifier was studied experimentally. A theoretical explanation was given by using the transmission matrix approach. The difference of nonlinearity in the two passbands was found to be enhanced greatly by the mechanism of asymmetric facet reflection.
\end{abstract}

Active optical bistability in Febry-Perot (FP) and distributed-feedback (DFB) semiconductor laser amplifiers has the advantages that the power required for switching is approximately three orders of magnitude smaller than that of the passive bistability and, at the same time, the optical gain of the active bistability can provide a sufficient optical power to drive the subsequent optical gates in the system. ${ }^{1,2}$ An additional advantage offered by the DFB laser amplifier bistable element is that its filter characteristic can dramatically reduce spontaneous emission ${ }^{3}$ in comparison with the FP laser amplifier, and a higher contrast of optical logical gate can be achieved. ${ }^{4}$ The two passbands of a DFB laser amplifier, which are determined by the Bragg diffraction, also make it possible for it to be used in systems with two different wavelengths.

In this Letter we extend the average field approximation reported in Refs. 1 and 2 to give a unified treatment of optical bistability for FP and DFB laser amplifiers and for a DFB laser amplifier with finite facet reflections. This particular structure is important in determining the single-longitudinal-mode behavior of DFB lasers and the filter characteristic of DFB laser amplifiers. Here we show, experimentally and theoretically, that this additional structure in a DFB laser amplifier will result in a large nonlinearity difference with respect to its two passbands.

The experimental setup is as follows. Two identical DFB buried-heterostructure laser diodes were used as a probe and an amplifier; both of them had one facet antireflection coated and the other facet cleaved. The lasing wavelengths were approximately $1554 \mathrm{~nm}$, and the probe was biased at 2.5 times its threshold current. Two diffraction-limited lenses with a N.A. of 0.6 were used for the beam coupling. An optical isolator inserted between the probe and the amplifier provided $40 \mathrm{~dB}$ of isolation, and a half-wavelength plate was used to match the polarization of the two lasers. A monochromator and a FP scanning interferometer were used for rough and fine measurement of the spectrum. Frequency matching and adjusting were accomplished by controlling the laser heat-sink temperature, and the change of the coupled optical power caused by the change of temperature was controlled to less than $5 \%$. Figure 1 gives the measured output near the two frequencies corresponding to the lasing band [Fig. 1(a)] and the nonlasing band [Fig. 1(b)] of the amplifier biased at $95 \%$ of its threshold. The probe power coupled into the amplifier was approximately $0.9 \mu \mathrm{W}$. The short-wavelength band (which would lase if biased above threshold) shows a bistability loop, whereas the long-wavelength band shows no bistability. When the input optical power was increased to 50 $\mu \mathrm{W}$, both bands show bistable output, as can be seen in Fig. 2. However, the loop widths are obviously different for the lasing band [Fig. 2(a)] and the nonlasing band [Fig. 2(b)]. Figure 3 gives the measured loop widths of bistability against the input optical power. Bistability occurs for the lasing band at an input power as low as $0.2 \mu \mathrm{W}$ and at approximately $10 \mu \mathrm{W}$ for the nonlasing band.

The nonlinearity difference in the two passbands of
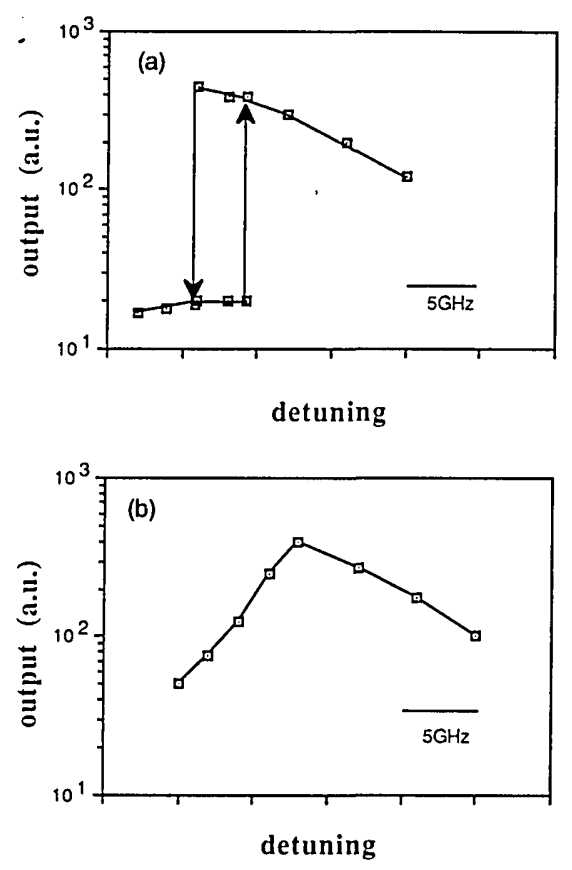

Fig. 1. Measured output of the amplifier versus frequency detuning with an input optical power of $0.9 \mu \mathrm{W}$ (a) for the lasing band and (b) for the nonlasing band. The amplifier was biased at $0.95 I_{\text {th }}$. 


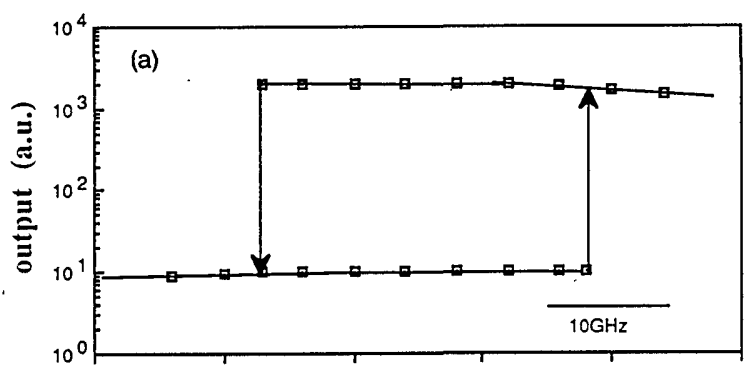

detuning

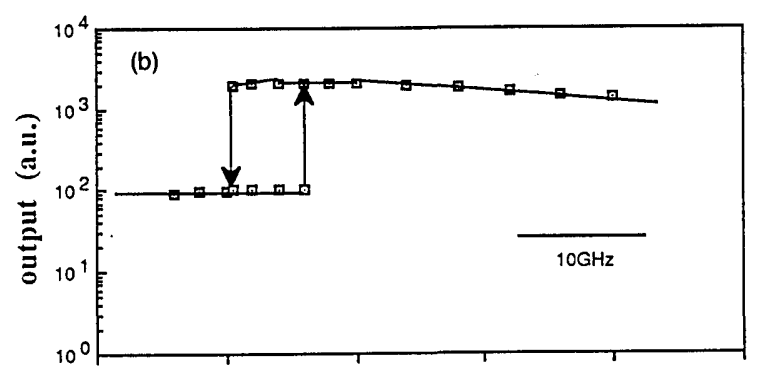

detuning

Fig. 2. Measured output of the amplifier versus frequency detuning with an input optical power of $50 \mu \mathrm{W}$ (a) for the lasing band and (b) for the nonlasing band. The amplifier was biased at $0.95 I_{\mathrm{th}}$. (The absolute vertical values are not calibrated.)

a DFB laser amplifier was first predicted by Adams and $W_{y a t t^{2}}$ for a semiconductor laser amplifier with an ideal DFB structure. However, they obtained a relatively small nonlinearity difference. Hence, the theory in Ref. 2 is not able to explain the above-mentioned experiment results in which the difference of the loop widths between the two bands is obvious. We believe that this nonlinearity difference enhancement is mainly caused by the laser amplifier's asymmetric facet reflection.

The starting point of our calculation is the standard coupled-mode equations for a DFB laser,

$$
\begin{aligned}
& \frac{\mathrm{d} A}{\mathrm{~d} z}=(g+i \delta) A+i_{\kappa} B, \\
& \frac{\mathrm{d} B}{\mathrm{~d} z}=-(g+i \delta) B-i_{\kappa} A,
\end{aligned}
$$

where $\kappa$ is the grating coupling coefficient, $g$ is the modal gain per unit length, $\delta$ is the detuning, and $A(z)$ and $B(z)$ are the complex amplitudes of the forward and backward propagating waves, respectively. In the mean field approximation, the solution of Eqs. (1) can be written in the transmission matrix form as

$$
\left[\begin{array}{l}
A(z) \\
B(z)
\end{array}\right]=\left[\begin{array}{ll}
F_{11} & F_{12} \\
F_{21} & F_{22}
\end{array}\right]\left[\begin{array}{l}
A\left(L^{-}\right) \\
B\left(L^{-}\right)
\end{array}\right],
$$

where

$$
\begin{aligned}
& F_{11}=\left[\exp (-\gamma x)-u^{2} \exp (\gamma x)\right] /\left(1-u^{2}\right) \\
& F_{22}=\left[\exp (\gamma x)-u^{2} \exp (-\gamma x)\right] /\left(1-u^{2}\right) \\
& F_{21}=-F_{12}=u[\exp (-\gamma x)-\exp (\gamma x)] /\left(1-u^{2}\right) \\
& \gamma= \pm\left[\kappa^{2}+(g+i \delta)^{2}\right]^{1 / 2}
\end{aligned}
$$

$$
u=i \kappa /[\gamma-(g+i \delta)] \text {, }
$$

with $x=L-z . \quad z=L^{-}$indicates the position located just at the left of $z=L$ inside the cavity, where $L$ is the cavity length. The parameter $u$ can be interpreted as the effective reflection of the grating, and the sign of $\gamma$ is chosen such that $|u(\gamma)| \leq 1,5$ so that the limit case of $\kappa=0$ corresponds to $u=0$. Using the boundary conditions $B\left(L^{-}\right)=r_{2} A\left(L^{-}\right)$and $A\left(L^{-}\right)=P_{0}^{1 / 2} /(1-$ $\left.r_{2}^{2}\right)^{1 / 2}$, where $P_{0}$ is the output power and $r_{2}$ is the amplitude reflectivity of the output facet, we get

$$
|A(z)|^{2}=P_{0}\left|F_{11}+r_{2} F_{12}\right|^{2} /\left(1-r_{2}^{2}\right)^{1 / 2}
$$

and

$$
|B(z)|^{2}=P_{0}\left|F_{21}+r_{2} F_{22}\right|^{2} /\left(1-r_{2}^{2}\right)^{1 / 2} .
$$

The average field intensity inside the active cavity is defined by $E_{\mathrm{av}}{ }^{2}=(1 / \mathrm{L}) \int_{0}^{L} \mid A(z) \exp (-i \beta z)+B(z)$ $\left.\exp (i \beta z)\right|^{2} \mathrm{~d} z$, where $\beta$ is the propagation constant. Here the cavity is considered to be much longer than the wavelength, so that when the cross terms of $A(z)$ and $B(z)$ are averaged to be zero, we obtain

$$
\begin{gathered}
E_{\mathrm{av}}^{2}=\frac{P_{0}}{\left|1-u^{2}\right|\left|1-r_{2}^{2}\right|}\left(\frac { 1 + | u | ^ { 2 } } { 2 \gamma _ { r } L } \left\{\left[1-\exp \left(-2 \gamma_{r} L\right)\right]\right.\right. \\
\left.\times\left|1+r_{2} u\right|^{2}-\left[1-\exp \left(2 \gamma_{r} L\right)\right]\left|u+r_{2}\right|\right\}-\frac{2}{\gamma_{r} L} \\
\left.\quad \times \operatorname{Re}\left[u\left(u+r_{2}\right)\left(1+u^{*} r_{2}{ }^{*}\right) \sin \left(2 \gamma_{i} L\right)\right]\right),
\end{gathered}
$$

where $\gamma_{r}=\operatorname{Re}(\gamma)$ and $\gamma_{i}=\operatorname{Im}(\gamma)$. In this expression, the reflectivity of the input facet $r_{1}$ does not appear, because $r_{1}$ is already involved in the relationship between $P_{0}$ and the input signal. The nonlinear properties are characterized by the mean field intensity-dependent detuning $\delta$ and modal gain $g$ as ${ }^{1}$

$$
\delta=\delta_{0}+\frac{\alpha g_{0} I_{\mathrm{av}} / I_{s}}{2\left(1+I_{\mathrm{av}} / I_{s}\right)} ; \quad g=\frac{g_{0}}{1+I_{\mathrm{av}} / I_{s}}-\alpha_{0},
$$

where $\alpha$ is the linewidth enhancement factor that represents the gain-induced index variation, $I_{s}$ is the saturation intensity of the material, $g_{0}$ and $\delta_{0}$ are the small-

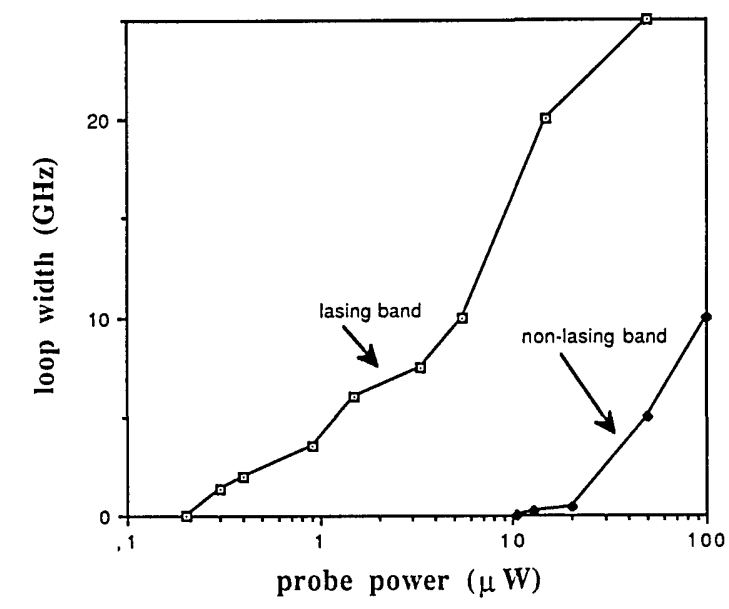

Fig. 3. Measured bistability loop width versus input optical power. The amplifier was biased at $0.95 I_{\text {th }}$. 

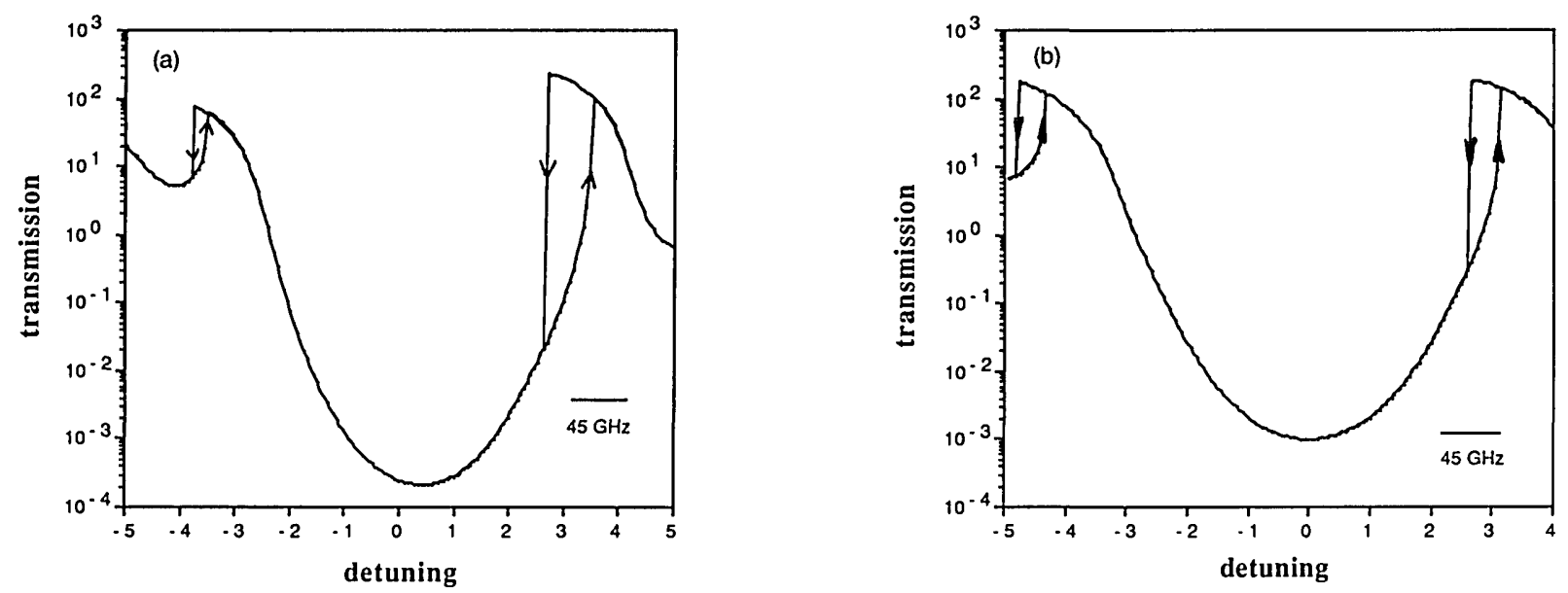

Fig. 4. Calculated transmission of a DFB laser amplifier versus detuning with respect to the Bragg wavelength. (a) With one facet antireflection coated and the other facet cleaved; $r_{1}=0, r_{2}=0.56$. (b) With both of the facets antireflection coated; $r_{1}=0$, $r_{2}=0$.

signal gain and detuning, respectively, and $\alpha_{0}$ is the cavity loss.

To relate both the output and input signals with the average field intensity, the reflections of both facets should be considered. The transmission matrix formulation leads to

$$
\begin{aligned}
{\left[\begin{array}{l}
E_{i} \\
E_{r}
\end{array}\right]=\frac{1}{\left(1-u^{2}\right) t_{1} t_{2}}\left[\begin{array}{rr}
1 & -r_{1} \\
-r_{1} & 1
\end{array}\right]\left[\begin{array}{ll}
F_{11} & F_{12} \\
F_{21} & F_{22}
\end{array}\right] } \\
\times\left[\begin{array}{ll}
1 & r_{2} \\
r_{2} & 1
\end{array}\right]\left[\begin{array}{l}
A\left(L^{+}\right) \\
B\left(L^{+}\right)
\end{array}\right],
\end{aligned}
$$

where $E_{i}$ and $E_{r}$ are the incident and reflected field amplitudes, respectively. The transmission and reflection characteristics of DFB laser amplifiers can then be obtained:

$$
\begin{aligned}
& T=\left|\frac{P_{0}}{E_{i}^{2}}\right|=\frac{\left|1-u^{2}\right|^{2}\left|\left(1-r_{1}^{2}\right)\left(1-r_{2}^{2}\right)\right|}{\left|\exp (-\gamma L)\left[1+u\left(r_{1}+r_{2}\right)+r_{1} r_{2} u\right]-\exp (\gamma L)\left[u^{2}+u\left(r_{1}+r_{2}\right)+r_{1} r_{2}\right]\right|^{2}}, \\
& R=\left|\frac{E_{r}}{E_{i}}\right|^{2}=\frac{\left|1-u^{2}\right|^{2}\left|\left(1-r_{1}^{2}\right)\left(1-r_{2}^{2}\right)\right|}{\mid \exp (\gamma L)\left[\left(1+u r_{1}\right)\left(u+r_{2}\right)\right]-\exp (-\gamma L)\left[\left.\left(u+r_{1}\right)\left(1+u r_{2}\right)\right|^{2}\right.} \cdot
\end{aligned}
$$

When the facets' reflections $r_{1}$ and $r_{2}$ are set to zero, Eqs. (4), (7), and (8) are reduced to Eqs. (5), (6), and (7) of Ref. 2. If, on the other hand, the coupling coefficient $\kappa$ is set to zero, with $\gamma=g+i \delta$ and $u=0$, Eqs. (4) and (8) are the same as Eqs. (2) and (1) of Ref. 1 , where the FP laser amplifier was considered.

However, it is the trade-off of DFB and facet reflections that enhances the nonlinearity difference in the two passbands. The results of a calculation including the facet reflection give a large gain difference for the two passbands, as shown in Fig. 4(a), which was plotted by using Eq. (7) with the parameters $\kappa=2.4, g_{0}=$ $0.95 g_{\text {th }}, P_{i}=0.0041 I_{s}, r_{1}=0, r_{2}=0.56$, and $\alpha=8.2$, where $g_{\text {th }}$ is the threshold gain and $P_{i}$ is the input optical power. This gives the explanation of the above experiment results. Note that in the absence of facet reflection, although there is some nonlinearity difference, the widths of the bistability loops of the two passbands are always similar, as shown in Fig. $4(b)$.

In conclusion, we have measured the difference of the bistability loops in the two passbands of a DFB semiconductor laser amplifier. A calculation was carried out for the bistability of a DFB semiconductor laser amplifier with a finite facet reflection. The results demonstrated that the difference of the bistable loop widths in the two passbands is enhanced greatly by the asymmetric facet reflection. The analysis is easily extended to study the nonlinear properties of a multisegment DFB laser amplifier.

The authors thank P. Spano and A. Mecozzi for helpful discussions. This research was carried out in the framework of the agreement between the Fonda- zione Ugo Bordoni and the Italian Post and Telecommunication Administration.

Rongqing Hui is on leave from Beijing University of Posts and Telecommunications, Beijing, China.

\section{References}

1. M. A. Adams, H. J. Westlake, M. J. O'Mahony, and I. D. Henning, IEEE J. Quantum Electron. QE-21, 1498 (1985).

2. M. A. Adams and R. Wyatt, IEE Proc. J. 134, 35 (1987).

3. K. Magari, S. Ishikawa, N. Takado, and K. Asakawa, IEEE J. Quantum Electron. 24, 2178 (1988).

4. W. F. Sharfin and M. Dagenais, Appl. Phys. Lett. 48, 1510 (1986).

5. G. P. Agrawal and N. K. Dutta, Long-Wavelength Semiconductor Lasers (Van Nostrand, New York, 1986), Chap. 7. 